\title{
EFFECTS OF SPEED AND INCLINE ON LOWER EXTREMITY KINEMATICS DURING TREADMILL JOGGING IN HEALTHY SUBJECTS
}

\author{
Lan-Yuen Guo ${ }^{1}$, Fong-Chin Su², Chich-Haung Yang ${ }^{3}$, Shu-Hui Wang ${ }^{3}$, Jyh-Jong Chang ${ }^{4}$, \\ WEN-LAN WU ${ }^{1}$, HWAI-TING LIN ${ }^{1}$ \\ ${ }^{1}$ Faculty of Sports Medicine, College of Medicine, Kaohsiung Medical University, \\ Kaohsiung, Taiwan \\ ${ }^{2}$ Institute of Biomedical Engineering, National Cheng Kung University, Tainan, Taiwan \\ ${ }^{3}$ Department of Physical Therapy, Tzu-Chi College of Technology, Hualien, Taiwan \\ ${ }^{4}$ Faculty of Occupation Therapy, College of Health Science, Kaohsiung Medical University, \\ Kaohsiung, Taiwan
}

\begin{abstract}
Recently, there are more people jogging with a treadmill at the gym or the home setting. The main available selected modes for treadmill jogging are speed and slope of incline. Increased speeds and incline slopes will not only increase the cardiopulmonary loading but may also alter the lower extremity (LE) movement patterns. There are few systematic investigations of the effect of the speed and incline on LE kinematics. Most studies have used 2D methods which focused on movements in sagittal plane only and this has limitations in the acquired data since lower extremity movements also include frontal and transverse planes. The current study aimed to investigate LE movement during jogging at different speeds and incline slopes using a high speed three-dimensional (3D) motion analysis system.

Eighteen young healthy males were recruited. The video-based motion capture system with six CCD cameras, HIRES Expert Vision System (Motion Analysis Corporation, CA, USA), was used to collect kinematic data at a sampling frequency of $120 \mathrm{~Hz}$. Nineteen passive reflective markers were attached to bilateral lower extremities of the subject. The joint angle is calculated by Euler angle

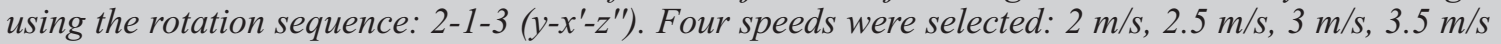
with the slope at 0 , and four slopes were selected: $0 \%, 5 \%, 10 \%, 15 \%$ at a speed of $3 \mathrm{~m} / \mathrm{s}$. Repeatedmeasures ANOVA was used to test hypotheses regarding changes in jogging condition on LE kinematic variables. The significance level was set at 0.05 .

As the jogging slope increased, the hip, knee and ankle demonstrated a significantly greater maximum flexion in swing phase $(p<0.001)$, but the maximum extension angles in stance phase were relatively unchanged. Increased LE flexion during swing phase is important to ensure foot clearance with increased slope. For increased speed, the hip and ankle joints had significantly greater maximum joint extension angles during stance phase and the hip and knee joint had significantly larger maximum flexion angles in swing phase $(p<0.001)$. Increased motion during swing phase account for a larger step length and increased motion during stance phase may facilitate the generation of power during forward propulsion as the jogging speed increased. As the slope and speed increased, LE movement patterns were changed in the transverse plane: the significantly increased $(p<0.01)$ internal hip rotation at terminal stance, the increased toe-in of foot $(p<0.001)$ during terminal stance phase and decreased $(p<0.05)$ toe-out during swing phase. Increased hip motion in transverse plane could lengthen the stride distance and increase foot toe-in for providing a stable lever for push off to increase propulsion force as speed or slope is increased. By way of systematic $3 D$ kinematic investigation of the $L E$ in jogging, the results further elucidate the effect of changing speed and incline on LE joints movements. This information could provide guidelines for rehabilitation clinicians or coaches to select an appropriate training mode for jogging.
\end{abstract}

Biomed Eng Appl Basis Comm, 2006(April); 18: 73-79.

Keywords: jogging, Biomechanics, incline, speed, kinematics, three-dimensional 


\section{INTRODUCTION}

Jogging is not only the most common movement for athletes during competitive sports, but also it is the most popular exercise for people to maintain their wellness. In the US, there are more than 30 million people take jogging as their usual exercise program for leisure or competitive purpose. However lower extremity injuries are quite prevalent and it is estimated that there is $1 / 2-1 / 4$ of frequent runners will sustain sports injuries of the lower extremity each year[1 ]. Biomechanical investigation in jogging activity is essential since the major reason for jogging injury is overuse problem during the long distance and long period. The estimated numbers for foot strike on the ground is nearly 500 600 steps for one-kilometer jogging. Jogging is a natural extension of walking[2]. During walking there is bilateral foot contact on the ground (double support phase). As walking speed is increased, double support will disappear and is replaced by bilateral foot off the ground (double float phase), which is defined as jogging. A complete gait cycle is the duration from one foot strike on the ground to the next foot strike. Clinicians and researchers have applied gait cycle to describe what occurs during this period. The foot-off the ground is about $60 \%$ of the gait cycle for walking and is shorten to about $40 \%$ for jogging[3]. Recently more people are taking up jogging with a treadmill at the gym or home setting. The main available modes that can be select for treadmill jogging are the speed and slope of incline.

Increased speed and incline slope will not only enlarge the cardiopulmonary loading[4-5] but also alter the movement pattern of lower extremity (LE) joints [6-9]. Previous studies on biomechanical investigations of jogging effect on LE is performed during level ground walking $[3,10-14]$ and most have used 2D methods. 2D kinematic analysis will only provide movement data for the sagittal plane and is limited in the examination of lower extremity movement for the frontal and transverse plane. As jogging speed increases, the knee will have increased joint motion[3] and maximum knee flexion in swing phase[12-14]. However the increased maximum knee flexion angle in swing phase is largely different with a study that report [8] 13 degrees and another [9] suggesting 28 degrees even through at similar speeds from $3 \mathrm{~m} / \mathrm{s}$ to $6 \mathrm{~m} / \mathrm{s}$.

Received: Dec. 10, 2005; Accepted: Feb. 7, 2006 Correspondence: Chich-Haung Yang, PT, MSc, PhD Candidate

Department of Physical Therapy, Tzu-Chi College of Technology, No.880, Sec. 2, Jianguo Rd., Hualien City, Hualien County 97005, Taiwan

E-mail: r.chyang@tccn.edu.tw
However, as jogging speed increased, changes in maximum knee flexion at stance phase is not consistent, one study that reported the angle is decreased [9] and another study found this increased $[8,14]$. Also, as the speed increased, decreased maximum knee extension at stance phase is noted [14]. Similarly, the hip increased but by only 3-5 degrees in maximum extension in stance phase as jogging speed dramatically increased from $3 \mathrm{~m} / \mathrm{s}$ to $6 \mathrm{~m} / \mathrm{s}$, as compared with maximum flexion increased by about 20 degrees in swing phase $[8,9,14]$. A slight increased ankle maximum dorsiflexion and plantarflexion with increased jogging speed is reported [8-9].

For slope jogging, it is usually used for resistance training and has been shown to improve runner performance. However, the degree of slope for training is still controversial since this may change the runner" $\mathrm{s}$ jogging mechanism. Therefore decreasing the training effect once is not specific to the usual level jogging condition. However, the kinematic investigation of jogging is quite limited, and only increased knee flexion and ankle dorsiflexion at foot strike are noted in literature[15].

The current study aimed to investigate LE movement during jogging at different speeds and incline slopes using a high speed $3 \mathrm{D}$ motion analysis system.

\section{METHOD}

Eighteen young healthy males were recruited in the experiment (mean age: 23.3 years, mean height: $173.8 \mathrm{~cm}$ and mean weight: $68.1 \mathrm{~kg}$ ). Participants were excluded if they had or currently have any LE neuromuscular disease (ex. poliomyelitis) or serious LE musculoskeletal injury (ex. fracture) history. The six-camera video-based motion capture system, HIRES Expert Vision System (Motion Analysis Corporation, CA, USA), was used to collect kinematics data at a sampling frequency of $120 \mathrm{~Hz}$ (Figure 1).

Nineteen passive reflective markers were attached to bilateral lower extremities of the subject. Markers were placed over bilateral ASIS, lateral and medial sides of the knee joint axis, lateral and medial malleolus, sacrum, bilateral heels, bony landmark between the second and third metatarsal heads, and attach wands on bilateral thighs, shanks (Figure 2). Anthropometric data, including height, body mass, leg length, thigh length, shank length, and foot length (with shoes), were all measured by a physical therapist. The anthropometric measures of all subjects and the attachment of markers were performed by the same physical therapist. The lower limb segments were assumed as rigid bodies. The forward direction of the 
laboratory was set as $\mathrm{x}$-axis and lateral direction was $y$-axis. The upward direction of the laboratory was z-axis. The joint angle was calculated by Euler angle using the rotation sequence: $2-1-3\left(y-x^{\prime}-z^{\prime \prime}\right)$.

All subjects had a 5 minute warm-up prior to testing by walking on Kistler Gaitway, a treadmill (HPMost Corporation) embedded two force plates (Kistler, Switzerland) at $1.6 \mathrm{~m} / \mathrm{s}$ initially. Four speeds were selected: $2 \mathrm{~m} / \mathrm{s}, 2.5 \mathrm{~m} / \mathrm{s}, 3 \mathrm{~m} / \mathrm{s}, 3.5 \mathrm{~m} / \mathrm{s}$ with the slope at 0 , and four slopes were selected: $0 \%, 5 \%, 10 \%, 15 \%$ at a speed of $3 \mathrm{~m} / \mathrm{s}$. Repeated-measures ANOVA with SAS version 8 software program was used to test hypothesis regarding changes in jogging condition on LE kinematics variables. The significance level was set at 0.05 .
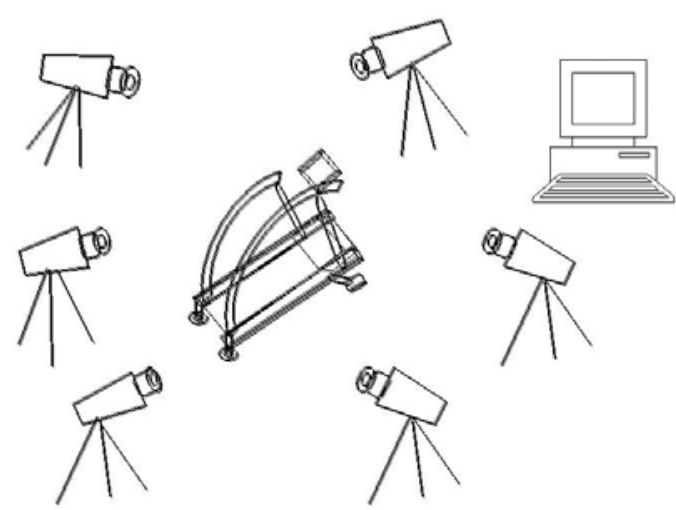

Fig 1. The Eva system, CCD cameras and Gaitway treadmill

(A)

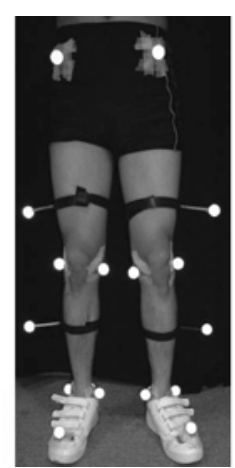

(B)

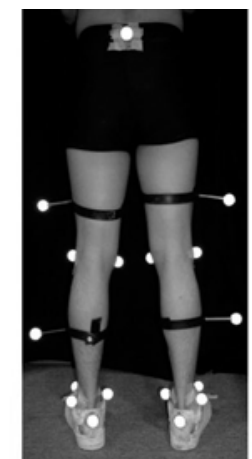

Fig 2. Marker setting for the lower extremity (A: front view; B: back view)

\section{RESULT}

Increasing slope during jogging resulted in earlier LE take-off the ground. The hip, knee and ankle demonstrated a significantly larger maximum flexion in swing phase as slope increased $(p<0.001$; Figure $3 \sim 5$, Table I ), but the maximum extension angles in stance phase were relatively unchanged. For increased speed, the hip and ankle joints had significantly $(p<0.001)$ increased maximum joint extension angles in stance phase and the hip and knee joint had significantly $(p<0.001)$ increased maximum flexion angles in swing phase (Figure 3 5, Table II).

As the slope increased, changes in LE movement pattern occurred in the transverse plane, with the hip showing significantly increased $(p<0.01)$ internal rotation during terminal stance, and increased toe-in $(p<0.001)$ at terminal stance and decreased toe-out
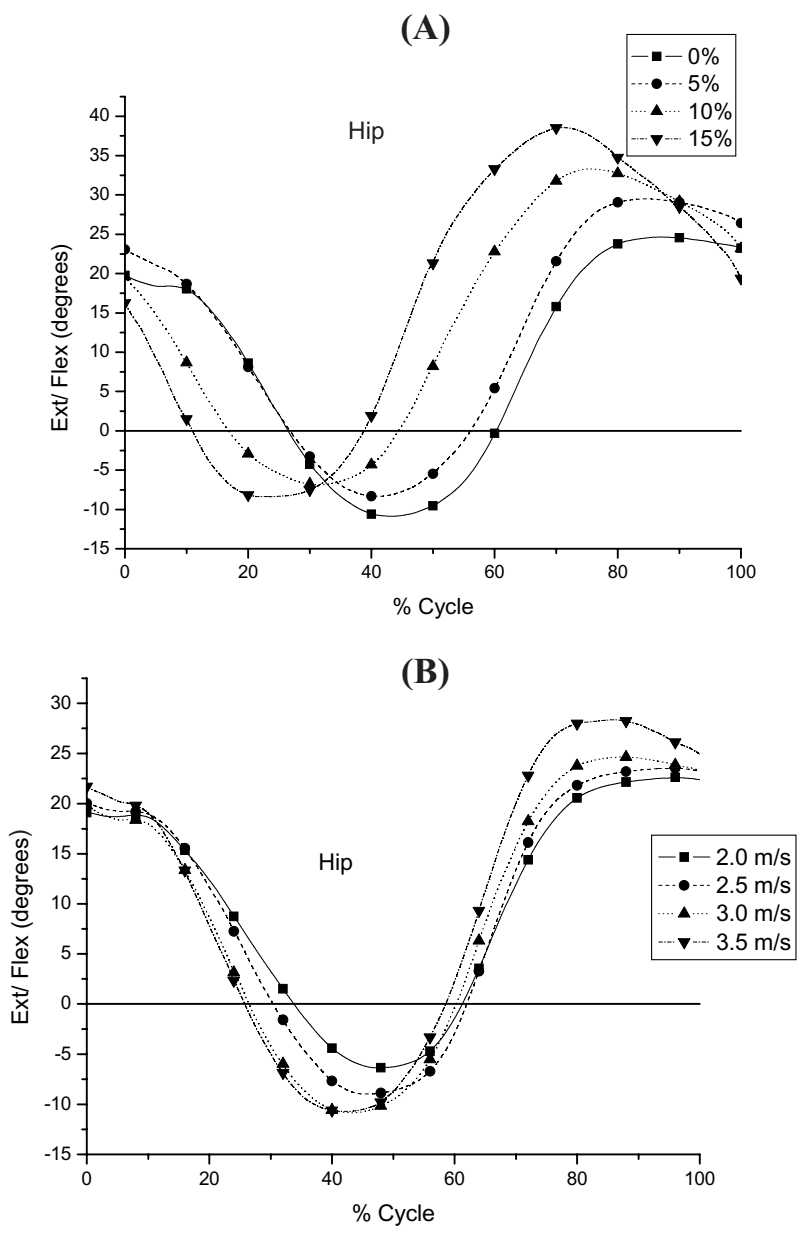

Fig 3. Hip joint motion in sagittal plane during jogging cycle with different incline (A) and speed (B) conditions. 

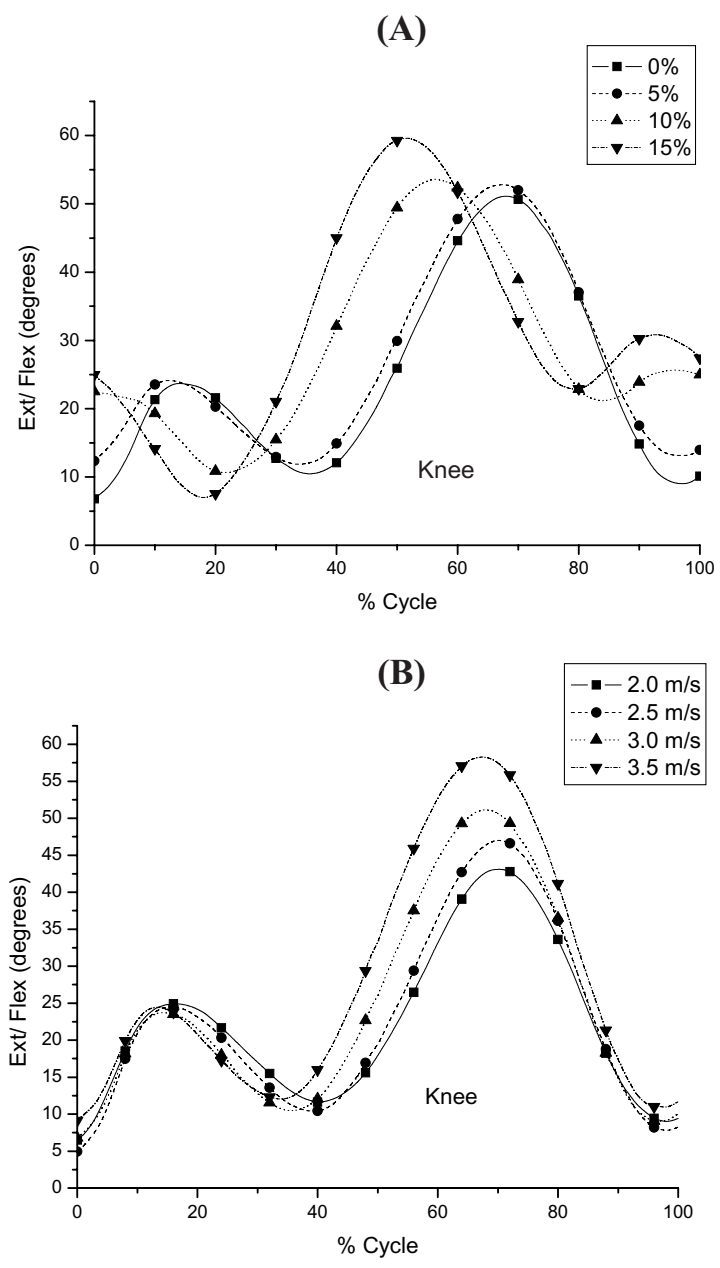

Fig 4. Knee joint motion in sagittal plane during jogging cycle with different incline (A) and speed (B) conditions.

$(\mathrm{p}<0.05)$ in swing phase at the foot (Figure 6-7, Table III). Similar changes were found for increased speed plus an increased hip external rotation at foot contact was noted $(p<0.01$, Figure $6-7$, Table IV). In the coronal plane, no significant differences were found for changing jogging conditions (Figure 8, Table IIIIV).

\section{DISCUSSION}

Despite the biomechanical analysis of jogging is not a new area of interest, the interest in it has prompted a comparable explosion of research by updated technical advances such as 3D motion analysis and faster cameras now. In the present study, we successfully applied a high speed 3D motion analysis system to systematically investigate the effect of the
(A)

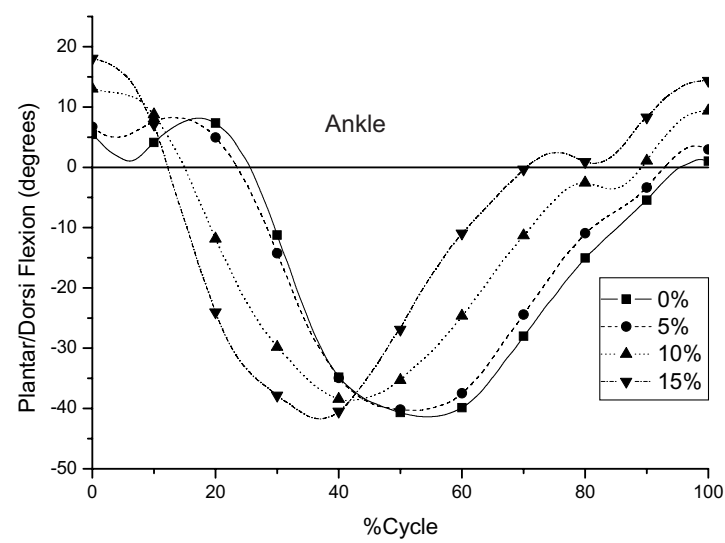

(B)

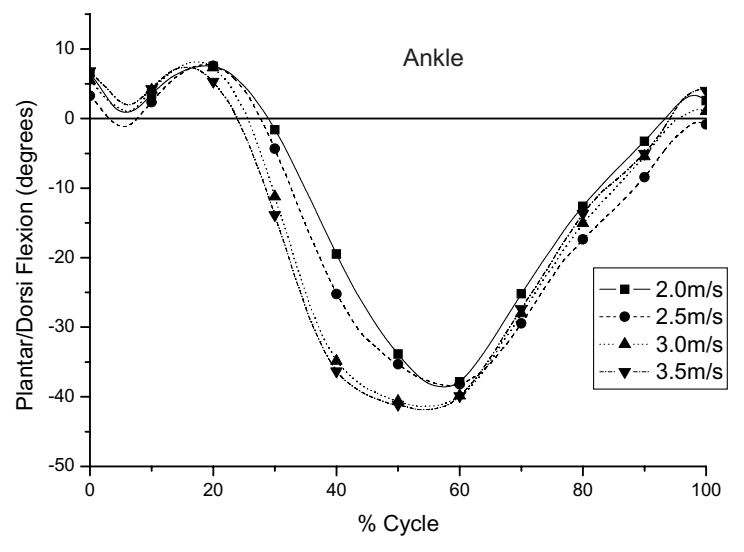

Fig 5. Ankle joint motion in sagittal plane during jogging cycle with different incline (A) and speed (B) conditions.

speed and incline jogging on LE kinematics. This information could fill the gap of the findings lacked in the previous related literature.

The hip demonstrated increased flexion during swing phase for a larger step length as jogging speed increased. As a consequence, earlier extension was noted at mid-swing for preparing landing in higher jogging speed. As jogging speed increased, the knee decreased flexion in early stance phase and increased flexion in swing phase. At early stance phase, knee movement had slight flexion and returned to extension (screw home mechanism), and is important for shock absorption. As the jogging speed was increased, the absorption ability may be decreased by shortened gait cycle as well as knee range of motion as observed in our findings. During swing phase, similar to increased hip flexion, increased knee flexion was observed with increased step length as required by increased jogging 
Table I. Peak values LE joint angles ${ }^{\dagger}$ in sagittal plane in different jogging slope condition

\begin{tabular}{cccccc}
\hline Degree & SL0 $^{\mathrm{b}}$ & SL5 & SL10 & SL15 & F value $^{\mathrm{c}}$ \\
\hline Hip Flex & 24.7 & 29.6 & 33.5 & 37.9 & $135.4^{* * *}$ \\
& $(5.2)$ & $(6.4)$ & $(6.1)$ & $(5.8)$ & \\
Hip Ext & 13.9 & 11.1 & 11.0 & 10.9 & $10.5^{* * *}$ \\
& $(4.7)$ & $(6.3)$ & $(6.2)$ & $(6.1)$ & \\
Knee Flex & 53.0 & 54.7 & 57.1 & 61.0 & $23.7 * * *$ \\
& $(10.5)$ & $(10.6)$ & $(11.9)$ & $(9.6)$ & \\
Knee Ext & -2.3 & -6.0 & -5.7 & -4.7 & $12.0 * * *$ \\
& $(7.7)$ & $(7.1)$ & $(6.4)$ & $(7.1)$ & \\
Ankle D/F & 12.2 & 12.7 & 16.0 & 20.0 & $63.6^{* * *}$ \\
& $(3.8)$ & $(4.9)$ & $(4.5)$ & $(4.7)$ & \\
Ankle P/F & 43.6 & 43.1 & 43.4 & 42.8 & 0.3 \\
& $(7.0)$ & $(7.5)$ & $(7.4)$ & $(7.0)$ & \\
\hline
\end{tabular}

$\dagger$ Means and SD in parenthesis

${ }^{a}$ Flex: flexion; Ext: extension

b SL0: slope 0\%; SL5: slope 5\%; SL10: slope 10\%; SL15: slope $15 \%$

'Statistical difference was analyzed with Repeated Measured ANOVA; ${ }^{*} \mathrm{p}<0.05 ;{ }^{*}{ }^{*} \mathrm{p}<0.01 ;{ }^{*} *{ }^{*} \mathrm{p}<0.001$

Table II. Peak values LE joint angles ${ }^{\dagger}$ in sagittal plane in different jogging speed condition

\begin{tabular}{cccccc}
\hline Degree & SP20 $^{\mathrm{b}}$ & SP25 & SP30 & SP35 & F value $^{\mathrm{c}}$ \\
\hline Hip Flex & 22.5 & 24.0 & 24.7 & 28.9 & $58.8^{* * *}$ \\
& $(5.9)$ & $(5.9)$ & $(5.2)$ & $(6.6)$ & \\
Hip Ext & 9.3 & 11.7 & 13.9 & 13.7 & $35.9^{* * *}$ \\
& $(6.5)$ & $(5.2)$ & $(4.7)$ & $(5.7)$ & \\
Knee Flex $^{\mathrm{a}}$ & 44.3 & 48.1 & 53.0 & 61.7 & $79.7^{* * *}$ \\
& $(7.4)$ & $(10.3)$ & $(10.5)$ & $(10.9)$ & \\
Knee Ext & -2.9 & -2.1 & -2.3 & -3.5 & $2.9 *$ \\
& $(6.9)$ & $(7.5)$ & $(7.7)$ & $(8.0)$ & \\
Ankle D/F & 11.8 & 12.1 & 12.2 & 13.1 & $2.9 *$ \\
& $(3.5)$ & $(4.3)$ & $(3.8)$ & $(4.6)$ & \\
Ankle P/F & 40.6 & 43.9 & 43.6 & 44.9 & $8.3 * * *$ \\
& $(8.6)$ & $(8.6)$ & $(7.0)$ & $(7.1)$ & \\
\hline
\end{tabular}

$\dagger$ Means and SD in parenthesis

${ }^{\text {a }}$ Flex: flexion; Ext: extension

b SP20: speed $2.0 \mathrm{~m} / \mathrm{s}$; SP25: speed $2.5 \mathrm{~m} / \mathrm{s}$; SP30: speed $3.0 \mathrm{~m} / \mathrm{s} ; \mathrm{SP} 35$ : speed $3.5 \mathrm{~m} / \mathrm{s}$

c Statistical difference was analyzed with Repeated Measured ANOVA; ${ }^{*} p<0.05 ;{ }^{*}{ }^{*} p<0.01 ; * * * p<0.001$

speed. The ankle demonstrated a more complicated pattern on initial contact as foot strike patterns changed from heel strike to toe strike, as increased jogging speed. As toe strike appeared during increased speed, the ankle will have decreased dorsiflexion at initial contact. However, as the speed is increased, the ankle has increased plantarflexion for push off, which can has generated more power for forward propulsion. Ankle dosiflexion in swing phase is limited as

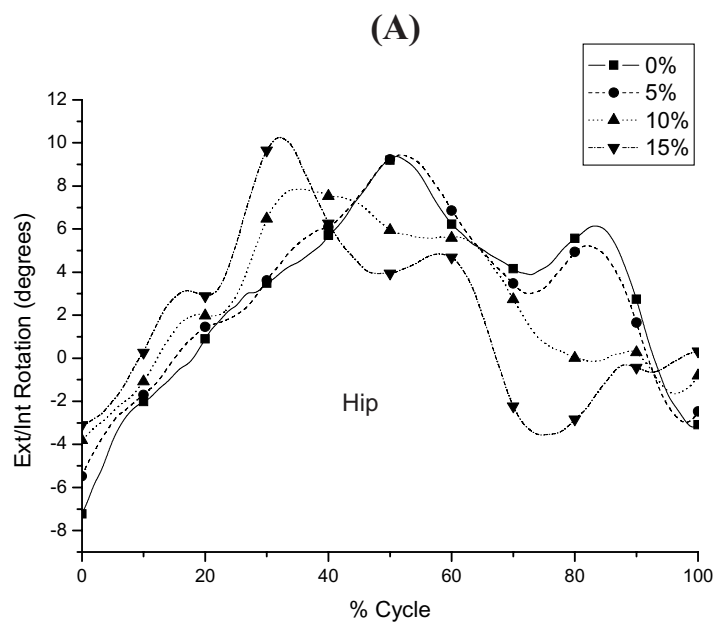

(B)

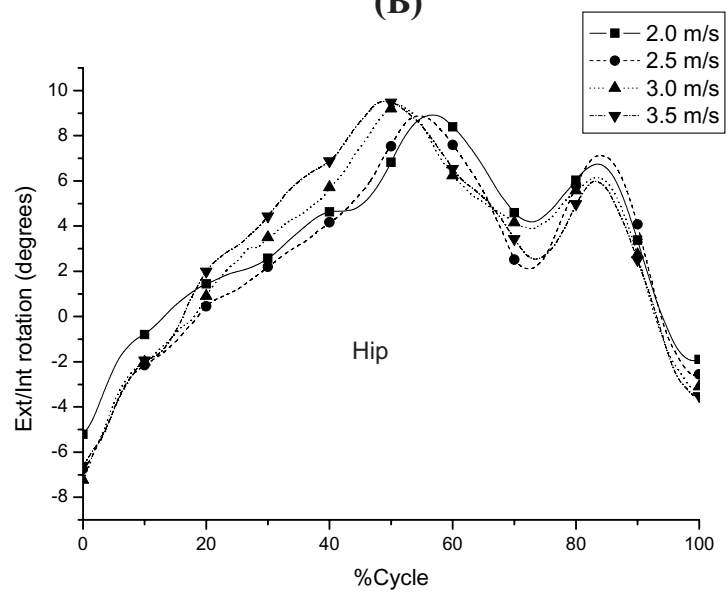

Fig 6. Hip joint motion in transverse plane during jogging cycle with different incline (A) and speed (B) conditions.

increases in jogging speed occurs since increased hip and knee flexion to overcome toe clearance such that further dorsiflexion may not be necessary. As jogging speed increased initially, except from $2.0 \mathrm{~m} / \mathrm{s}$ to 2.5 $\mathrm{m} / \mathrm{s}$, increases of hip maximum extension in stance phase is greater than increased maximum flexion in swing phase. However, while speed increased from $3.0 \mathrm{~m} / \mathrm{s}$ to $3.5 \mathrm{~m} / \mathrm{s}$, the pattern is reversed and increases in maximum hip extension in stance phase is lesser than increase of the maximum flexion in swing phase. The trend is consistent with previous literature that examined this when jogging speed was dramatically increased from $3 \mathrm{~m} / \mathrm{s}$ to $6 \mathrm{~m} / \mathrm{s}[8,9]$. That increased slope in jogging has increased resistance for a runner will increase the propulsion effort for the same jogging speed. Increased hip, knee and ankle flexion in swing and early stance phase is important to ensure foot clearance and heel contact as jogging at increased 
(A)

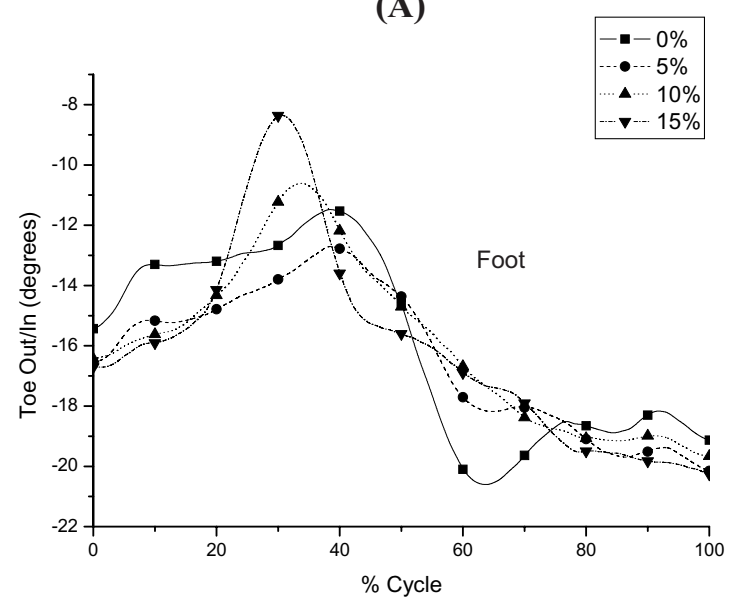

(B)

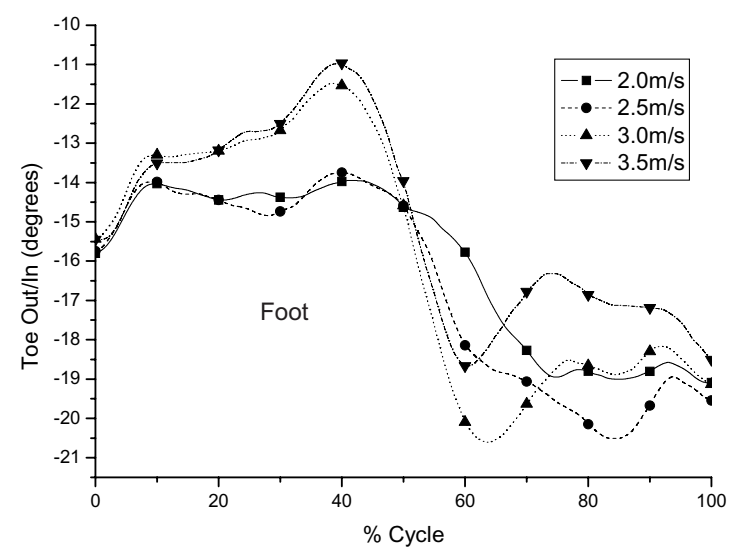

Fig 7. Foot progression angle during jogging cycle with different incline (A) and speed (B) conditions.

slope.

Besides the change in sagittal plane, the results suggest different jogging condition could vary LE movement especially in transverse plane. As the speed or slope of incline increased, LE changed its movement pattern in the transverse plane, with the hip showing increased hip external rotation at foot contact and increased internal rotation at terminal stance, as well as the foot showing increased toe-in at terminal stance and decreased toe-out in swing phase. Movement of the hip with pelvic movements in the transverse plane could lengthen the stride distance. Increased foot toe-in is accompanied with subtalar inversion for providing a stable lever for push off as required for increased propulsion force as increased speed or slope.

Systematic 3D kinematic investigations of the speed and incline settings on recreational treadmills
Table III: Peak values LE joint angles ${ }^{\dagger}$ in frontal and transverse plane in different jogging slope condition

\begin{tabular}{cccccc}
\hline Degree & SL0 $^{\mathrm{b}}$ & SL5 & SL10 & SL15 & F value $^{\mathrm{c}}$ \\
\hline Hip Abd $^{\mathrm{a}}$ & 15.0 & 14.8 & 14.6 & 15.3 & 1.98 \\
& $(3.2)$ & $(2.9)$ & $(3.4)$ & $(3.2)$ & \\
Hip Add & -3.5 & -4.3 & -3.7 & -3.3 & 1.53 \\
& $(1.7)$ & $(3.2)$ & $(3.4)$ & $(3.8)$ & \\
Hip IR & 11.2 & 10.7 & 12.4 & 13.4 & $6.19^{* *}$ \\
& $(6.9)$ & $(7.4)$ & $(7.4)$ & $(8.4)$ & \\
Hip ER & 9.5 & 8.6 & 8.8 & 10.0 & 1.86 \\
& $(5.6)$ & $(6.9)$ & $(6.3)$ & $(7.2)$ & \\
Foot Toe/In & -8.1 & -9.0 & -7.1 & -5.6 & $8.65^{* * *}$ \\
& $(4.6)$ & $(5.6)$ & $(7.4)$ & $(7.0)$ & \\
Foot Toe/Out & 21.9 & 21.2 & 20.6 & 20.0 & $4.0 *$ \\
& $(7.6)$ & $(6.5)$ & $(6.0)$ & $(5.5)$ & \\
\hline
\end{tabular}

$\dagger$ Means and SD in parenthesis

aAbd: Abduction; Add: Adduction; IR: Internal Rotation; ER: External Rotation

bSL0: slope 0\%; SL5: slope 5\%; SL10: slope 10\%; SL15: slope $15 \%$

'Statistical difference was analyzed with Repeated Measured ANOVA; ${ }^{*} \mathrm{p}<0.05 ;{ }^{*} \mathrm{p}<0.01 ; * * * \mathrm{p}<0.001$

Table IV. Peak values LE joint angles ${ }^{\dagger}$ in frontal and transverse planes in different jogging speed condition

\begin{tabular}{cccccc}
\hline Degree & SP20 & SP25 & SP30 & SP35 & F value $^{\mathrm{c}}$ \\
\hline Hip Abd & 15.0 & 14.9 & 15.0 & 15.4 & 0.8 \\
& $(3.1)$ & $(2.8)$ & $(3.2)$ & $(3.7)$ & \\
Hip Add & 4.6 & 3.6 & 3.5 & 4.0 & 1.4 \\
& $(3.3)$ & $(2.0)$ & $(1.7)$ & $(3.7)$ & \\
Hip IR & 9.8 & 9.7 & 11.2 & 11.6 & $5.1^{* *}$ \\
& $(6.7)$ & $(7.0)$ & $(6.9)$ & $(7.0)$ & \\
Hip ER & 7.5 & 9.5 & 9.5 & 9.5 & $5.6^{* *}$ \\
& $(5.5)$ & $(4.8)$ & $(5.6)$ & $(6.0)$ & \\
Foot Toe/In & -9.2 & -8.8 & -8.1 & -6.0 & $6.7^{* * *}$ \\
& $(5.4)$ & $(6.7)$ & $(4.6)$ & $(6.1)$ & \\
Foot Toe/Out & 21.1 & 22.7 & 21.9 & 20.6 & $3.9^{*}$ \\
& $(7.3)$ & $(8.2)$ & $(7.6)$ & $(7.6)$ & \\
\hline
\end{tabular}

$\dagger$ Means and SD in parenthesis

aAbd: Abduction; Add: Adduction; IR: Internal Rotation; ER: External Rotation

bSP20: speed $2.0 \mathrm{~m} / \mathrm{s}$; SP25: speed $2.5 \mathrm{~m} / \mathrm{s}$; SP30: speed $3.0 \mathrm{~m} / \mathrm{s} ;$ SP35: speed $3.5 \mathrm{~m} / \mathrm{s}$

'Statistical difference was analyzed with Repeated Measured ANOVA; ${ }^{*} \mathrm{p}<0.05 ; * * \mathrm{p}<0.01 ; * * * \mathrm{p}<0.001$

show that LE joint motions are affected differently for each setting. This information could provide rehabilitation clinicians or athletic coaches with guidelines for selecting appropriate modes for jogging. 
(A)

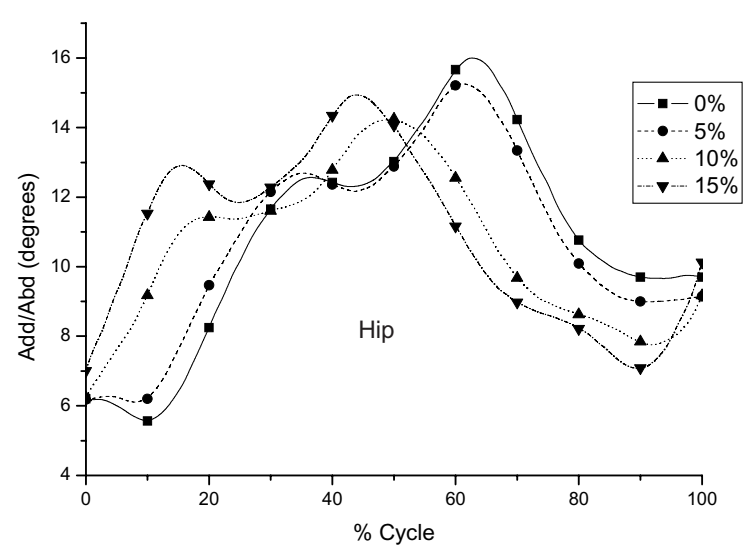

(B)

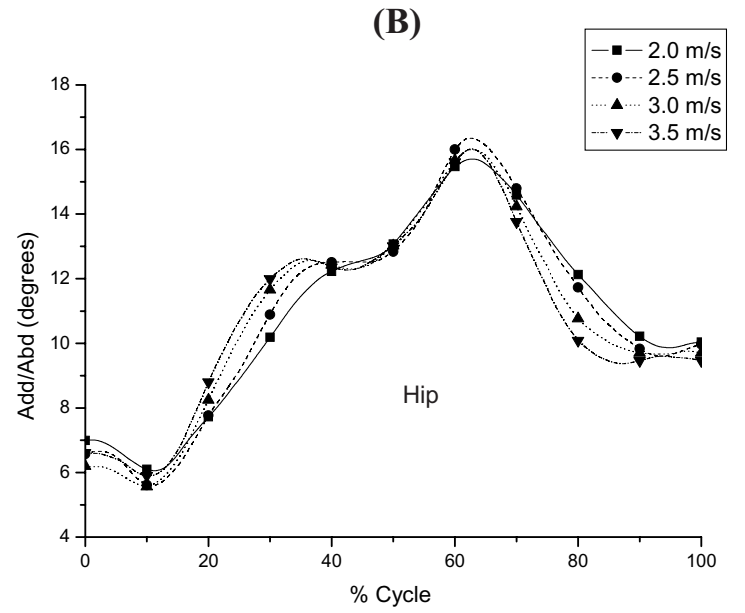

Fig 8. Hip joint motion in coronal plane during jogging cycle with different incline (A) and speed (B) conditions.

\section{ACKNOWLEDGEMENT}

This work is supported by Nation Science Council NSC92-2320-B-277-003, Taiwan.

\section{REFERENCE}

1. Clement, DB and Taunton, JE, A guide to the prevention of running injuries. Aust Fam Physician 1981;10:156-64.

2. Ounpuu, S, The biomechanics of walking and running. Clin Sport Med 1994;13:843-63.

3. Mann, RA and Hagy, J, Biomechanics of walking, running, and sprinting. Am J Sports Med 1980;8:345-50.
4. Doherty, M, Smith, PM, Hughes, MG, and Collins, $\mathrm{D}$, Rating of perceived exertion during highintensity treadmill running. Med Sci Sport Exer 2001;33:1953-8.

5. Klein, RM, Potteiger, JA, and Zebas, CJ, Metabolic and biomechanical variables of two incline conditions during distance running. Med Sci Sport Exer 1997;29:1625-30.

6. Swanson, SC and Caldwell, GE, An integrated biomechanical analysis of high speed incline and level treadmill running. Med Sci Sport Exer 2000;32:1146-55.

7. Grillner, S, Halbertsma, J, Nilsson, J, and Thorstensson, A, The adaption to speed in human locomotion. Brain Res 1979;165:177-82.

8. Sinning, WE and Forsyth, HL, Lower-limb actions while running at different velocities. Med Sci Sports 1970;2:28-34.

9. Nilsson, J, Thorstensson, A, and Halbertsma, J, Changes in leg movements and muscle activity with speed of locomotion and mode of progression in humans. Acta Physiol Scand 1985;123:457-75.

10. Winter, DA, Moments of force and mechanical power in jogging. J Biomech 1983;16:91-7.

11. Chapman, AE and Caldwell, GE, Factors determining changes in lower limb energy during swing in treadmill running. J Biomech 1983;16:6977.

12. Novacheck, TF, Walking, running, and sprinting: A three-dimensional analysis of kinematics and kinetics. Instr Course Lect 1995;44:497-506.

13. Novacheck, TF, The biomechanics of running. Gait Posture 1998;7:77-95.

14. Miller, DI, Biomechanics of running-what should the future hold? Can J Sport Sci 1978;3:229-36.

15. Hamill, CL, Clarke, TE, Frederick, EC, Goodyear, LJ, and Howley, ET, Effects of grade running on kinematics and impact force. Med Sci Sport Exer 1984;16:185. 